\title{
MEASUREMENT OF RADIO EMISSION FROM EXTENSIVE AIR SHOWERS
}

\author{
JÖRG R. HÖRANDEL \\ Radboud University Nijmegen, Department of Astrophysics, \\ P.O. Box 9010, 6500 GL Nijmegen, The Netherlands \\ E-mail: j.horandel@astro.ru.nl, http://particle.astro.kun.nl
}

\begin{abstract}
A new promising development in astroparticle physics is to measure the radio emission from extensive air showers. The particles in the cascade emit synchrotron radiation $(30-90 \mathrm{MHz})$ which is detected with arrays of dipole antennas. Recent experimental efforts are discussed.
\end{abstract}

Keywords: astroparticle physics; cosmic rays; air showers; radio emission.

\section{Introduction}

An intense branch of astroparticle physics is the study of high-energy cosmic rays to reveal their origin, as well as their acceleration and propagation mechanisms. ${ }^{1-3}$ At energies exceeding $10^{14} \mathrm{eV}$ cosmic rays are usually studied by indirect measurements - the investigation of extensive air showers initiated by cosmic particles in the atmosphere. Different techniques are applied, like the measurements of particle densities and energies at ground level, or the observation of Čerenkov and fluorescence light. An alternative technique has been recently revitalized - the detection of radio emission (at tens of $\mathrm{MHz}$ ) from extensive air showers at energies exceeding $10^{16} \mathrm{eV}$.

Radio emission from air showers was experimentally discovered in 1965 at a frequency of $44 \mathrm{MHz} .{ }^{4}$ The early activities in the 1960s and 1970s are summarized in Ref. ${ }^{5}$ Only recently, fast analog-to-digital converters and modern computer technology made a clear detection of radio emission from air showers possible. LOPES, a LOFAR Prototype Station had shown that radio emission from air showers can be detected even in an environment with relatively strong radio frequency interference (RFI). ${ }^{6}$ Further investigations of the radio emission followed with LOPES $^{7-11}$ and the CODALEMA experiment, ${ }^{12,13}$ paving the way for this new detection technique. ${ }^{14}$

Most likely, the dominant emission mechanism of the radio waves in 
the atmosphere is synchrotron radiation due to the deflection of charged particles in the Earth magnetic field (geosynchrotron radiation). ${ }^{15-17}$ In the frequency range of interest $(30-90 \mathrm{MHz})$ the wavelength of the radiation is large compared to the size of the emission region: the typical thickness of the shower disc is about 1 to $2 \mathrm{~m}$ only. Thus, coherent emission is expected, which yields relatively strong signals (of the order of a few $\mu \mathrm{V} / \mathrm{m} \mathrm{MHz}$ ) at ground level.

Goal of the present activities is to further push the development of radio detection to become a new, independent way to measure the properties of air showers. Ultimate goal is to derive information about the primary, shower-inducing particle from the measurements, such as the energy and mass of the particle as well as the particle direction and point of incidence. An advantage of radio detection, e.g. with respect to the fluorescence technique, is that showers can be observed with almost $100 \%$ duty cycle.

The arrival direction can be inferred from the arrival time of the wavefront at the antennas. The measured radio signal at a certain distance from the shower axis is a good estimator for the number of particles at shower maximum, being almost independent of the primary particle type. ${ }^{18}$ This can be used to determine the shower energy. The curvature of the shower front has been investigated. ${ }^{19,20}$ It could be shown that the radius of curvature measured at ground level is related to the distance to the shower maximum. The depth of the shower maximum in the atmosphere $X_{\max }$ is an important observable to determine the mass of the primary particle. The study indicates a resolution of $X_{\max }$ from radio observations of order of $30-40 \mathrm{~g} / \mathrm{cm}^{2}$, i.e. comparable to the accuracy of present fluorescence detectors.

\section{Experimental Set-Ups}

\subsection{LOPES}

The LOPES experiment registers radio signals in the frequency range from 40 to $80 \mathrm{MHz} .{ }^{21}$ In this band are few strong man made radio transmitters only, the emission from air showers is still strong (it decreases with frequency), and background emission from the Galactic plane is still low. An active short dipole has been chosen as antenna. An inverted V-shaped dipole is positioned about $1 / 4$ of the shortest wavelength above an aluminum ground plate. In this way a broad directional beam pattern is obtained. LOPES comprises 30 antennas ${ }^{22}$ located on site of the KASCADEGrande experiment. ${ }^{23,24}$ The LOPES data acquisition is triggered by large 
air showers registered with KASCADE-Grande. The latter measures the showers simultaneously to LOPES and delivers precise information on the shower parameters, such as shower energy as well as position and inclination of the shower axis.

\subsection{CODALEMA}

The CODALEMA experiment is set up at the Nancay radio observatory. ${ }^{12,25}$ In the first phase six conic logarithmic periodic antennas of the Decametric array have been used. The present set-up comprises new antennas, developed to optimize simplicity, costs, and performance. ${ }^{26}$ The broadband antennas $(100 \mathrm{kHz}-220 \mathrm{MHz})$ are based on a fat active dipole concept. They are simple dipoles $(2 \times 0.6 \mathrm{~m}$ long $)$ placed $1 \mathrm{~m}$ above ground. 21 dipoles are oriented in east-west polarization direction and three dipoles in north-south direction. The set-up is completed by 17 plastic scintillation counters to measure the properties of air showers.

\subsection{LOFAR}

The Low Frequency Array (LOFAR) is a new digital radio observatory, presently under construction. ${ }^{27,28}$ An objective of LOFAR is the detection of radio emission from particle cascades, originating from extremely highenergy particles from outer space. Two main lines of research are followed: (i) the measurement of radio emission from extensive air showers, generated by interactions of high-energy cosmic rays in the atmosphere ${ }^{29}$ and (ii) the detection of radio emission of particle cascades in the Moon, originating from ultra high-energy neutrinos and cosmic rays interacting with the lunar surface. ${ }^{30}$

More than 40 stations with fields of relatively simple antennas will work together as digital radio interferometer. The antenna fields are distributed over several countries in Europe with a dense core in the Netherlands. The latter will have at least 18 stations in an area measuring roughly $2 \times 3 \mathrm{~km}^{2}$. Each station will comprise 96 low band antennas, simple inverted V-shaped dipoles (like the LOPES antennas), operating in the frequency range from 30 to $80 \mathrm{MHz}$. Each antenna will have a dipole oriented in north-south and east-west directions, respectively. In addition, fields a of high-band antennas will cover the frequency range from 110 to $240 \mathrm{MHz}$. For air shower observations the signals from the low band antennas are digitized

aThe fields comprise 48 antennas in the Dutch stations and 96 in the European ones. 
and stored in a ring buffer (transient buffer board, TBB). For valid triggers the data are send to a central processing facility, based on an IBM Blue Gene supercomputer. First data from LOFAR are expected in early 2010.

A small air shower array will be installed in the LOFAR core. It comprises 20 scintillator counters, each with an area of about $1 \mathrm{~m}^{2}$, the detectors have been used previously in the KASCADE experiment. ${ }^{23}$ The array will provide basic air shower information. It may also serve as trigger for the radio antennas.

\subsection{AERA - Pierre Auger Observatory}

Radio emission from air showers at the Pierre Auger Observatory will be measured with the Auger Engineering Radio Array (AERA). ${ }^{31}$ AERA is co-located with the infill array of the Auger Observatory and in the field of view of the high-altitude fluorescence telescopes (HEAT). ${ }^{32}$ This unique setup will allow to register air showers simultaneously with three independent detection methods: radio waves, fluorescence light, and particle detection in water Čerenkov detectors. AERA will comprise about 150 antennas located on an area of about $20 \mathrm{~km}^{2}$ and is designed to cover the energy range from $10^{17}$ to $10^{19} \mathrm{eV}$. During the first phase (Spring 2010) 24 logarithmic periodic dipole antennas will be deployed and put into operation.

Presently, different antenna designs are tested and optimized at the Auger site. Among them are logarithmic periodic dipole antennas ${ }^{33}$ and simple dipoles, as in the CODALEMA experiment. ${ }^{34}$ A ring antenna design and FPGA-based hardware for a self trigger algorithm are developed within LOPES $^{\text {STAR }}$ and at the Auger site. ${ }^{35-37}$

\subsection{Calibration}

The antenna gain pattern is typically obtained from numerical calculations. Two methods are available for the absolute calibration of the electronics. (i) In LOPES all antennas, including the complete analog electronics chain, have been individually calibrated with a reference radio source. ${ }^{38}$ (ii) An independent way is to use the galactic radio background as calibration source. The expected strength of the galactic radio signal can be calculated as function of siderial time as well as day of the year and can be used as reference. This technique is presently explored with AERA. ${ }^{39}$ The measured radio signal as function of siderial time is depicted in Fig. 1. The colors represent measurements at different days of the year. The siderial variations are clearly visible, illustrating the good sensitivity of the system. 


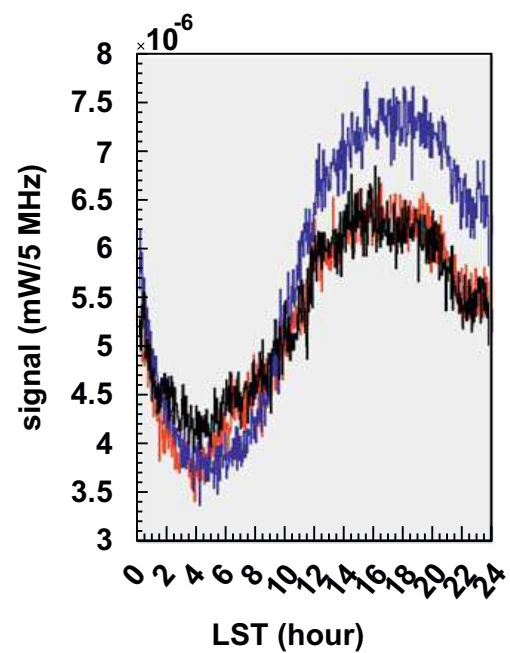

Fig. 1. Radio background measured at components as a function of azimuth angle the Auger Observatory as function of (top) and geomagnetic angle (bottom) as measiderial time. ${ }^{39}$
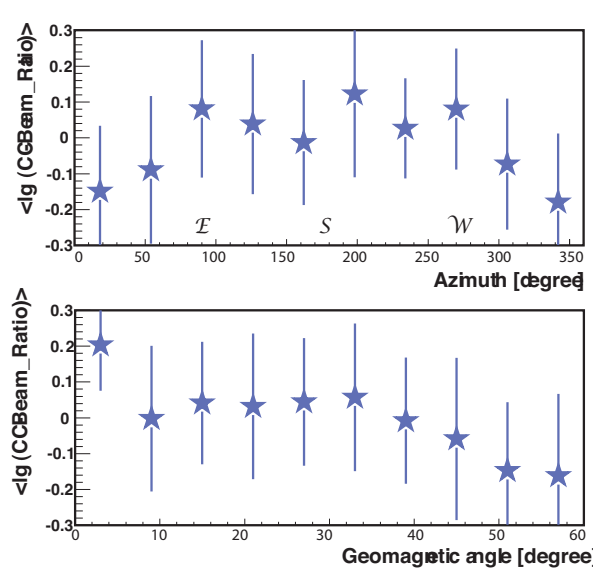

Fig. 2. Ratio of the measured signal strength of the north-south and east-west polarization sured by LOPES. ${ }^{40}$

\section{Radio Signal and Shower Parameters}

The properties of the showers registered by LOPES are simultaneously measured by the KASCADE-Grande air shower experiment. An empirical relation has been found to express the expected east-west component of the field strength at a distance $R$ from the shower axis as a function of shower parameters $^{41}$

$$
\begin{array}{r}
\epsilon=(11 \pm 1)[(1.16 \pm 0.025)-\cos \alpha] \cos \Theta \exp \left(\frac{-R}{(236 \pm 81) \mathrm{m}}\right) \\
\left(\frac{E_{0}}{10^{17} \mathrm{eV}}\right)^{(0.95 \pm 0.04)}\left[\frac{\mu \mathrm{V}}{\mathrm{m} \mathrm{MHz}}\right] .
\end{array}
$$

$\alpha$ is the angle between the shower axis and the direction of the Earth magnetic field (geomagnetic angle), $\Theta$ the zenith angle of the shower, and $E_{0}$ the energy of the shower inducing primary particle. It is interesting to note the absolute values of some parameters: the exponential fall-off has a characteristic length of about $240 \mathrm{~m}$, much larger than the classical Molière radius of electrons in air $(\approx 80 \mathrm{~m})$. This indicates that the lateral distribution of the radio component is flatter as compared to the electromagnetic component, an important fact to build large-scale radio arrays with an economic antenna density. The measured radio signal is almost directly proportional 
to the shower energy $\left(\propto E_{0}^{\approx 1}\right)$. Such a behavior is expected for a coherent emission of the radio waves in the air showers. This calibration of the measured radio signal is a first important step towards the application of the radio detection as independent method to register extensive air showers.

Recently, the lateral distribution of the measured radio signals has been investigated in more detail with LOPES. ${ }^{42,43}$ It is found that some air showers exhibit a relatively flat lateral distribution. Most likely, such events are registered at a small distance to the shower axis for showers with large zenith angles.

Also the CODALEMA group has parameterized the expected radio signals as function of shower parameters: ${ }^{44}$

$$
\begin{array}{r}
\epsilon=2.3 \frac{\mu \mathrm{V}}{\mathrm{m} \mathrm{MHz}} \frac{\Delta \nu}{\mathrm{MHz}} \frac{-\vec{v} \times \vec{B}}{|\vec{v}||\vec{B}|} \frac{|\vec{B}|}{47 \mu \mathrm{T}} \cos \Theta \exp \left(\frac{-R}{D_{0}(\Theta, \nu)}\right) \\
\frac{E_{0}}{10^{17} \mathrm{eV}}\left[\frac{\mu \mathrm{V}}{\mathrm{m}}\right] .
\end{array}
$$

With the parameters $\Delta \nu$ the frequency interval, energy $E_{0}$ and zenith angle $\Theta$ of the shower, $\vec{v}$ the direction of the shower axis, $\vec{B}$ the Earth magnetic field, $R$ the distance to the shower axis, and $D_{0}(\Theta, \nu)$ an empirical function. b

The frequency spectra in air showers have been investigated with both, the LOPES $^{10}$ and CODALEMA ${ }^{13}$ experiments, yielding similar results. Due to the relative small frequency range covered $(\approx 40-80 \mathrm{MHz})$ it can not be decided yet whether the data are described better by a power law or an exponential function.

\section{Polarization and Asymmetries}

The investigation of the polarization properties and of asymmetries in the arrival direction is the key to confirm the geosynchrotron mechanism and is currently the hottest topic discussed by CODALEMA and LOPES. The field strength produced near the motion axis of the particle is expected to be approximately proportional to $\vec{v} \times \vec{B}$ and the radiation is expected to be linearly polarized. ${ }^{45,46}$ The E-W and N-S polarization components are obtained by the projection of the $\vec{v} \times \vec{B}$ vector on the corresponding axis.

b The angle between $\vec{v}$ and $\vec{B}$ in (2) is $\alpha$ in (1). The $\alpha$ dependence in (1) and (2) is $(\approx 1)-\cos \alpha$ and $|\vec{v} \times \vec{B}| /(|\vec{v}||\vec{B}|)=\sin \alpha$, respectively. In the interval $[0, \pi / 2]$, both expressions yield similar values, which are compatible with the measurements, given the present accuracy of the data. 

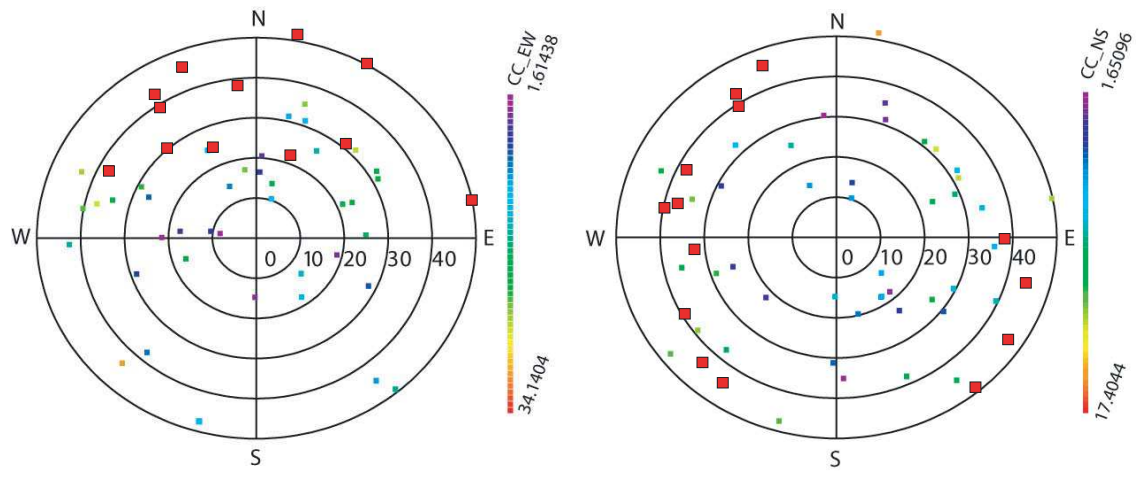

Fig. 3. Sky map of air showers detected in east-west (left) and north-south (right) polarization direction with LOPES. ${ }^{47}$ The boxes mark the arrival direction of the air showers with the strongest radio signal.

Strong radio signals are expected from showers with arrival directions being about perpendicular to the magnetic field in the respective polarization direction.

Since the Earth magnetic field is inclined ${ }^{\mathrm{c}}$ we expect an asymmetry in the observed signal strength on the sky. Sky maps of the E-W component as measured by the different experiments are shown in Fig. 3 (left) ${ }^{47}$ Fig. 4 (left), ${ }^{46}$ and Fig. $5 .{ }^{34}$ The color code in Fig. 3 represents the measured field strengths. The boxes indicate the directions of the events with the strongest signals. LOPES and CODALEMA clearly see more strong events from the north, while more strong events are registered from the south in the southern hemisphere. A similar effect is observed for the N-S component. In this case, strong events arrive from the east and the west, ${ }^{45,47}$ see Fig. 3 (right).

The ratio of the measured field strength in N-S to E-W polarization directions as measured by LOPES is depicted as function of azimuth (top) and geomagnetic angle (bottom) in Fig. 2. ${ }^{40,47}$ Mean values and the spread of the distributions are shown. A correlation between the plotted quantities can be recognized. The ratio of the north-south divided by the east-west component of the $\vec{v} \times \vec{B}$ vector of the measured showers exhibits the same behavior as a function of the azimuth angle and geomagnetic angle.

In addition, the CODALEMA group investigated the polarity of the fields measured at ground. ${ }^{45}$ Also in this observable clear asymmetries are

${ }^{\mathrm{c}}$ Elevation angles of the magnetic field are LOPES: $65^{\circ}$, CODALEMA: $63^{\circ}$, Auger: $-35^{\circ}$. 

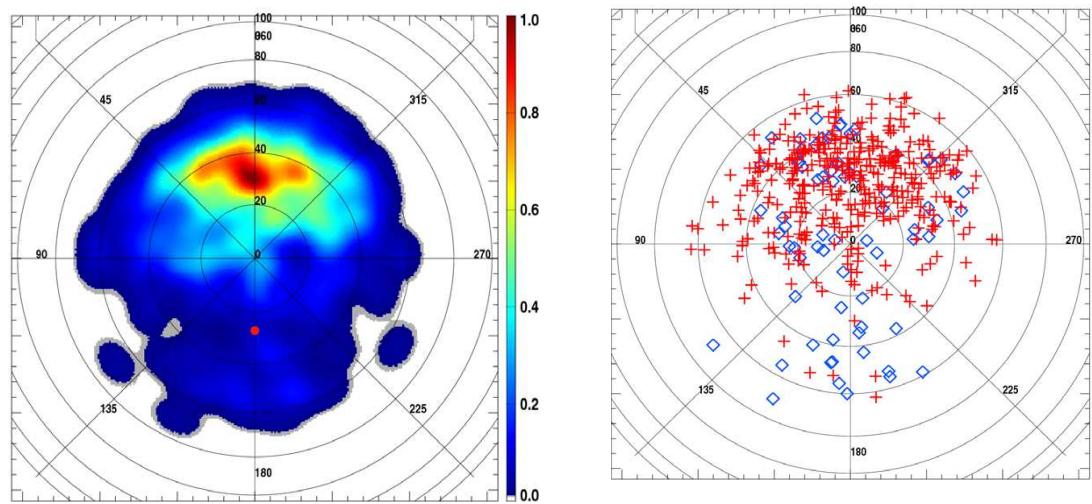

Fig. 4. Sky maps of events recorded with CODALEMA in E-W polarization direction. ${ }^{46}$ Left: arrival direction of showers. Right: polarity of measured signal.

measured. Showers with a positive electric field arrive mainly from the north and the east, while showers which induce a negative field preferably come from southern and western directions. Simulations of the radio emission by the CODALEMA group show the same behavior as the measured data for both polarization directions, for the arrival direction of the showers as well as for the polarity. ${ }^{45}$

The recent measurements and simulations conducted at various sites strongly support an emission process in air showers being proportional to $\vec{v} \times \vec{B}$ and they support geosynchrotron radiation as dominant process.

\section{Thunderstorm Events}

During thunderstorms there are strong (static) electric fields in the atmosphere which severely influence the radio emission of air showers. The study of radio emission during thunderstorms has a two-fold goal: (i) the study of the thunderstorms itself and (ii) the (early) detection of thunderstorm conditions to prevent false air shower reconstruction during thunderstorms.

As an example, a thunderstorm observed at the Auger Observatory is shown in Fig. $6{ }^{34}$ It can be recognized how the thunderstorm moves on the sky as function of time.

Quantitative investigations of the radio signals during thunderstorms have been conducted with LOPES data. ${ }^{9,43,48}$ The electric fields in the atmosphere yield to an acceleration of charged particles, which in turn yields to an amplification of the radio emission from air showers during 


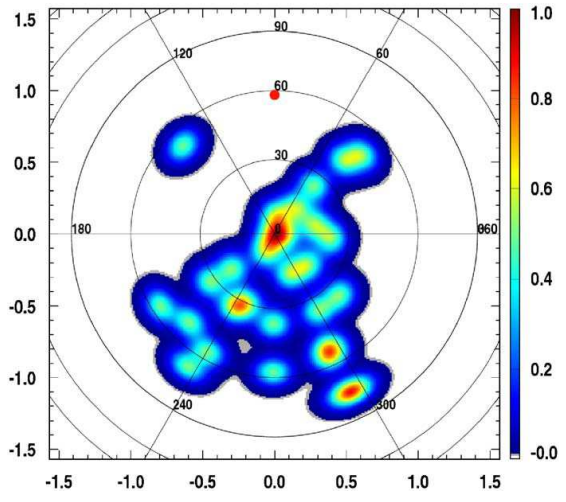

Fig. 5. Sky map of radio events detected at the Auger Observatory in E-W polarization direction. ${ }^{34}$

thunder storm conditions.

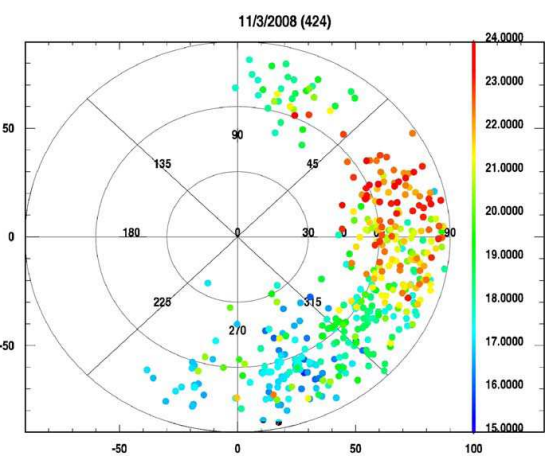

Fig. 6. Sky map of radio events detected during a thunderstorm at the Pierre Auger Observatory. The colors represent the observation time. ${ }^{34}$

\section{Outlook}

The investigations of radio signals from air showers with LOPES and CODALEMA are the basis for the application of these technique in large-scale experiments. The next step is to utilize the radio detection technique in large arrays, comprising several hundreds of antennas. The radio detection of air showers is a fast growing sub-discipline in astroparticle physics. With the new experiments starting operation, exciting results are expected in the next few years.

\section{Acknowledgments}

I would like to thank the conference organizers for their invitation to give this review in the inspiring environment of Lago di Como and the Villa Olmo. I'm thankful to my colleagues from LOPES, LOFAR, and the Pierre Auger Observatory for fruitful discussions.

\section{References}

1. J. Blümer, R. Engel and J. Hörandel, Prog. Part. Nucl. Phys. 63, p. 293 (2009).

2. J. Hörandel, Adv. Space Res. 41, p. 442 (2008).

3. J. Hörandel, Rev. Mod. Astron. 20, p. 203 (2008).

4. J. Jelley et al., Nature 205, p. 327 (1965). 
5. H. Allan, Progress in Elementary Particles and Cosmic Ray Physics (J.G. Wilson \& S.G. Wouthuysen eds., North Holland, 1971), p. 169.

6. H. Falcke et al., Nature 435, p. 313 (2005).

7. W. Apel et al., Astropart. Phys. 26, p. 332 (2006).

8. J. Petrovic et al., Astron. $E$ Astroph. 462, p. 389 (2007).

9. S. Buitink et al., Astron. \& Astroph. 467, p. 385 (2007).

10. A. Nigl et al., Astron. E Astroph. 488, p. 807 (2008).

11. A. Nigl et al., Astron. ES Astroph. 487, p. 781 (2008).

12. D. Ardouin et al., Nucl. Instr. \& Meth. A 555, p. 148 (2005).

13. D. Ardouin et al., Astropart. Phys. 26, 341 (2006).

14. H. Falcke et al. arXiv:0804.0548, (2008).

15. T. Huege and H. Falcke, Astron. ES Astroph. 412, p. 19 (2003).

16. T. Huege and H. Falcke, Astron. Es Astroph. 430, p. 779 (2005).

17. T. Huege and H. Falcke, Astropart. Phys. 24, p. 116 (2005).

18. T. Huege et al., Astropart. Phys. 30, p. 96 (2008).

19. S. Lafèbre et al. submitted to Astropart. Phys., (2008).

20. S. Lafèbre et al., Proc. 31th Int. Cosmic Ray Conf., Lodz, \# 587 (2009).

21. A. Horneffer et al., Proc. of the SPIE 5500, p. 129 (2004).

22. S. Nehls et al., Proc. 29th Int. Cosmic Ray Conf., Pune 8, p. 45 (2005).

23. T. Antoni et al., Nucl. Instr. $\&$ Meth. A 513, p. 490 (2003).

24. G. Navarra et al., Nucl. Instr. ES Meth. A 518, p. 207 (2004).

25. O. Ravel et al., Nucl. Instrum. Meth. A518, 213 (2004).

26. D. Ardouin et al., Nucl. Instrum. Meth. A572, 481 (2007).

27. LOFAR. www.lofar.org, (2009).

28. J. Hörandel et al., Nucl. Phys. B (Proc. Suppl.), in press (2009).

29. A. Horneffer et al., Proc. 31th Int. Cosmic Ray Conf., Lodz, \# 369 (2009).

30. K. Singh et al., Proc. 31th Int. Cosmic Ray Conf., Lodz, \# 1077 (2009).

31. A. van den Berg et al., Proc. 31th Int. Cosmic Ray Conf., Lodz, \# 232 (2009).

32. M. Kleifges et al., Proc. 31th Int. Cosmic Ray Conf., Lodz, \# 410 (2009).

33. S. Fliescher et al., Nucl. Instr. \& Meth. A 604, p. S225 (2009).

34. B. Revenu et al., Nucl. Instr. E Meth. A 604, p. S37 (2009).

35. H. Gemmeke et al. (Acoustic and Radio EeV Neutrino Detection Activities, R. Nahnhauer, S. Böser eds., World Scientific, 2005), p. 242.

36. O. Krömer et al., Proc. 31th Int. Cosmic Ray Conf., Lodz, \# 1232 (2009).

37. A. Schmidt et al., Proc. 31th Int. Cosmic Ray Conf., Lodz, \# 1124 (2009).

38. S. Nehls et al., Nucl. Instrum. Meth. A589, 350 (2008).

39. J. Coppens et al., Nucl. Instr. \& Meth. A 604, p. S41 (2009).

40. P. Isar, Proc. 31th Int. Cosmic Ray Conf., Lodz, \# 1128 (2009).

41. A. Horneffer et al., Proc. 30th Int. Cosmic Ray Conf., Merida 4, p. 83 (2008).

42. S. Nehls et al., Proc. 31th Int. Cosmic Ray Conf., Lodz, \# 417 (2009).

43. J. Hörandel et al., these proceedings, (2009).

44. C. Riviere, private communication, (2009).

45. C. Riviere et al., arXiv:0906.2720, (2009).

46. D. Ardouin et al., Astropart. Phys. 31, 192 (2009).

47. P. Isar et al., Nucl. Instr. \& Meth. A 604, p. S81 (2009).

48. M. Ender et al., Proc. 31th Int. Cosmic Ray Conf., Lodz, \# 405 (2009). 